Diabetologe 2021 · 17:481

https://doi.org/10.1007/s11428-021-00781-5

Angenommen: 27. Mai 2021

(c) Springer Medizin Verlag $\mathrm{GmbH}$, ein Teil von Springer Nature 2021

Das Diabetes Update 2021 in Mainz wurde wegen der weiter anhaltenden COVID-19-Pandemie (COVID-19: „Coronavirus disease 2019“) nun schon zum 2. Mal als Livestream präsentiert, auch dieses Mal wieder mit aktiver Einschaltung der Zuhörer. Innerhalb der 2 Tage der Veranstaltung mit 14 Spezialvorträgen wurden mehr als 260 konkrete Fragen zu den einzelnen Themenblöcken gestellt, von denen ein Großteil von den Referenten schon innerhalb der Veranstaltung beantwortet werden konnte. Hieran wurde wieder einmal die Exzellenz der Experten auf ihrem Gebiet ersichtlich.

Zwischen der ersten Insulinbehandlung eines Diabetes und den heutigen Möflichkeiten der Diabetestherapie liegen Welten

Vor inzwischen 100 Jahren wurde der erste Patient mit Diabetes mit dem lebensrettenden Insulin behandelt. Wer hätte sich damals vorstellen können, dass solche Menschen heute ein weitgehend normales Leben führen können, und wer hätte gedacht, dass einmal das Hauptaugenmerk nicht mehr auf dem reinen Überleben und der Verhinderung von Akutkomplikationen des insulinpflichtigen Diabetes, sondern auf der Vermeidung von Langzeitfolgen liegt, und dass eine bestimmte Diabetesform, der Typ-2Diabetes, einmal ein epidemisches Ausmaß erreichen würde, und dass die kontinuierliche Messung des Glukosespiegels einmal durch ein Gerät übernommen werden kann, und dass selbst die Verabreichung von Insulin z. T. von Apparaten übernommen wird, und, und, und ...

\title{
Werner Scherbaum
}

Heinrich-Heine-Universität Düsseldorf, Universitätsklinikum, Düsseldorf, Deutschland

\section{Diabetes Update 2021}

In diesem Heft sollen die Neuerungen zu 5 Themen des Diabetes Update 2021 von den Referenten ausführlich dargestellt werden:

- Screening und Epidemiologie (Prof. Wolfgang Rathmann, Deutsches

Diabeteszentrum Düsseldorf)

- Typ-2-Diabetes (Prof. Andreas Hamann, Hochtaunusklinik Bad Homburg)

- Diabetische Nephropathie und Hypertonie (Prof. Tom Lindner, Universitätsklinikum Leipzig)

- Gastroenterologie und Hepatologie bei Diabetes (Prof. Jörg Bojunga, Universitätsklinikum Frankfurt)

Das aus meiner Sicht Wichtigste zu den anderen Vorträgen soll im Folgenden in aller Kürze aufgezeigt werden, wobei zu erwähnen ist, dass die besten Anregungen aus der Teilnahme an der gesamten Veranstaltung resultieren.

Den Leitern des Diabetes Update 2021, den Herren Prof. Andreas Hamann, Dr. Helmut Kleinwechter, Prof.
Stefan Martin und Prof. Michael Stumvoll sowie dem Team von MedUpdate danke ich ausdrücklich für ihre große Mühe bei der Organisation der gelungenen Veranstaltung, welche mir selbst und sicherlich auch allen anderen Teilnehmern viele neue Erkenntnisse und Anregungen für die eigene Praxis gab.

In Vorfreude auf das Diabetes Update 2022 verbleibe ich

Ihr

Werner Scherbaum

\section{Korrespondenzadresse}

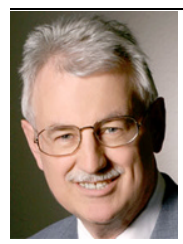

Univ.-Prof. Dr. med. Werner Scherbaum Heinrich-Heine-Universität Düsseldorf, Universitätsklinikum

Moorenstraße 5, 40225 Düsseldorf, Deutschland scherbaum@ uni-duesseldorf.de

Interessenkonflikt. W. Scherbaum gibt an, dass kein Interessenkonflikt besteht.

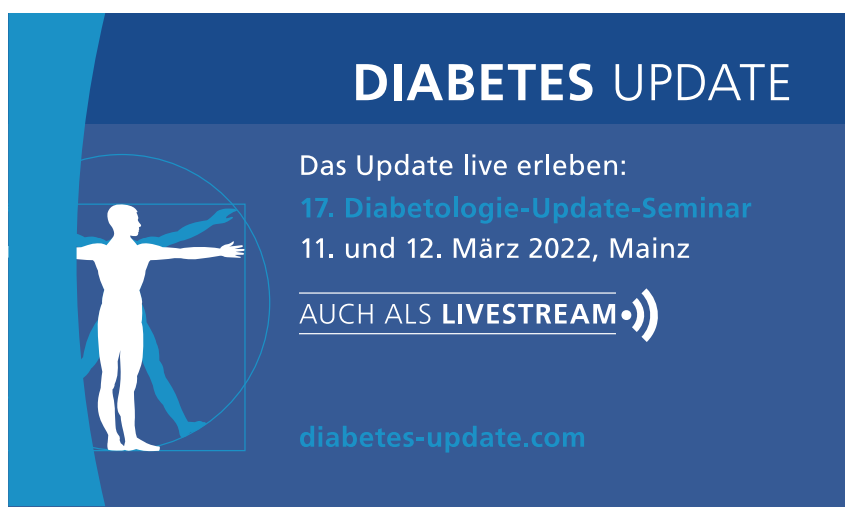

